\title{
Estrategias de comunicación para mejorar el posicionamiento de la cooperativa de ahorro y crédito "San Jorge" Ltda.
}

Communication strategies to improve the positioning of the savings and credit cooperative "San Jorge” Ltda.

Denise Liliana Pazmiño Garzón. ${ }^{1}$, Yolanda Patricia Moncayo Sánchez. ${ }^{2}$, Marco Vinicio Carrión Torres. ${ }^{3} \&$ Víctor Hugo Cuadrado Samaniego. ${ }^{4}$

\begin{abstract}
.
DOI: https://doi.org/10.33262/cienciadigital.v3i3.3.769

The main objective of this research is to improve the positioning level of the savings and credit cooperative "San Jorge" Ltda. in Riobamba city, based on the diagnosis in which have been considered decision aspects valued by current and potentials customers of the cooperative. To achieve this objective, the research includes the main theories, theoretical approaches related to positioning and communication that support the proposal; regarding the methodological framework, a qualitative and quantitative approach was used, a descriptive research was applied based on synthetic, inductive and deductive analytical methods. The results obtained in the research determine the preferences of the population under study around the criteria considered when choosing a financial institution, which highlights solvency, security and trust, the low level of knowledge of the institution, the probability of being interested in the products and services offered by the cooperative, the main means of communication used; findings

\footnotetext{
${ }^{1}$ Escuela Superior Politécnica de Chimborazo, Facultad de Administración de Empresas. Riobamba, Ecuador. denise.pazmino@espoch.edu.ec

${ }^{2}$ Escuela Superior Politécnica de Chimborazo, Facultad de Administración de Empresas Riobamba, Ecuador. yolanda.moncayo@espoch.edu.ec

${ }^{3}$ Universidad de las Fuerzas Armadas, Riobamba, Ecuador. mvct1980@hotmail.com

4 Licenciado en comunicación social con especialidad en Desarrollo. Magíster en Gestión Pública. victorcuadrado81@gmail.com
} 
that constitute the input for the design of communication strategies, their execution contributed positive results, evidenced in the increase of the captures of partners and customers, placements of consumer credit and microcredit, knowledge and brand recall.

Keywords: Strategies, communication, positioning, cooperative, customers.

\section{Resumen}

La presente investigación tiene como objetivo fundamental mejorar el nivel de posicionamiento de la Cooperativa de Ahorro y Crédito "San Jorge" Ltda., en la ciudad de Riobamba, a partir del diagnóstico en el que se han contemplado aspectos de decisión valorados por los clientes actuales y potenciales de la cooperativa. Para el logro de dicho objetivo, la investigación recoge las principales teorías, enfoques teóricos relacionados al posicionamiento y comunicación que permiten sustentar la propuesta; respecto al marco metodológico se empleó un enfoque cuali- cuantitativo, se aplicó una investigación de tipo descriptivo apoyada en los métodos analítico sintético, inductivo y deductivo. Los resultados obtenidos en la investigación determinan las preferencias de la población objeto de estudio en torno a los criterios considerados al elegir una institución financiera en los que se destacan la solvencia, seguridad y confianza, el bajo nivel de conocimiento de la institución, probabilidad de interesarse en los productos y servicios ofertados por la cooperativa, principales medios de comunicación utilizados; hallazgos que constituyen el insumo para el diseño de las estrategias de comunicación, su ejecución aportaron resultados positivos, evidenciados en el incremento de las captaciones de socios y clientes, colocaciones de crédito de consumo y microcrédito, conocimiento y recordación de marca.

Palabras claves: Estrategias, comunicación, posicionamiento, cooperativa, clientes.

\section{Introducción}

En torno a la alta competitividad existente en el mercado, es prioritario darse a conocer, "vender" su actividad, establecer estrategias de Marketing, si se quiere permanecer en el mercado o recibir la aprobación de la sociedad, un plan de comunicación permitirá trazar una línea de propósitos que determina el cómo se pretende lograr los objetivos. Esta imperiosa necesidad de comunicar, de transmitir al exterior y al interior de la institución, debe hacerse de forma metódica y sistemática, a través de una correcta planificación y tras un previo análisis de aquellas circunstancias del entorno que pudieran influir en el proceso.

La comunicación corporativa es un importante elemento estratégico para todas las organizaciones a fin de lograr los objetivos. Puede ser definido como el conjunto de recursos de comunicación de los que dispone una empresa para intentar llegar a su público. 
ISSN: 2602-8085

Por consiguiente, cualquier empresa por pequeña que sea debe ser consciente que todo comunica, ya que no sólo transmite los anuncios publicitarios o las relaciones públicas, sino también la actividad cotidiana de la empresa, desde sus productos y servicios hasta el comportamiento de sus miembros.

Este entorno empresarial y la necesidad de mejorar el nivel de posicionamiento de la Cooperativa de Ahorro y Crédito "San Jorge" Ltda., es lo que ha impulsado a realizar la actual investigación.

La comunicación es dinámica, compleja y continuamente cambiante, por lo tanto, cuando la comunicación es social, implica a personas que interactúan con diferentes dimensiones, motivaciones y habilidades. La naturaleza del proceso de la comunicación también supone que pueden pasar muchas cosas desde el principio hasta el final de una conversación, donde las personas pueden cambiar mucho sus posiciones una vez que la conversación comienza (West \& Turner, 2005).

Para Bartolie (2013) se llama comunicación estratégica a la coordinación de todos los recursos comunicacionales externos e internos de la empresa (publicidad, marketing, folletería, canales comunicativos, ambiente laboral, organigrama, distribución espacial, higiene, atención al cliente, protocolo y ceremonial, posventa, etc.) para diferenciarlas diferentes competencias y lograr un lugar en la mente de los públicos que le interesa a la organización.

Por otro lado, Regouby (2014) determina que comunicación estratégica es el sistema de influencias psico-sociológicas destinadas a uno o por lo general varios públicos, a través de un conjunto relativamente amplio de acciones y medios de comunicación, durante un período de tiempo previamente establecido, persiguiendo objetivos en correspondencia con los intereses e intenciones de una determinada organización.

Kotler (2012), en otra instancia muestra una visión específica de la estrategia de comunicación al exponer que tiene el objetivo de difundir un grupo de sucesos de una organización para elaborar marcos de referencia que sean comunes entre los que emiten el mensaje y los que lo reciben. Bajo este esquema el estratega concibe a la comunicación como un proceso integrador y con un flujo de información bidireccional. Forma parte de un equipo de trabajo que escucha la opinión de otros para la toma de decisiones.

De acuerdo con Orejuela (2011), los elementos necesarios en una estrategia de comunicación está orientado establecer una línea de trabajo especializada que permita difundir a través de las diferentes herramientas atribuidas al ámbito de la difusión y comunicación, su imagen, sus principios y valores, sus contenidos y sus avances de manera sistemática. Una estrategia de comunicación debe ser un elemento imprescindible que debe ser desarrollado en varios pasos:

1. Análisis para determinar cuál es la situación presente de la comunicación. 
2. Determinar objetivos y alcance, planificar acciones, definir los mecanismos y metodología para llevar a cabo las acciones, y establecer la evaluación a lo largo del proceso.

3. Tener el compromiso de todas las partes intervinientes en las acciones que sean programadas, sensibilizar a los participantes y predisponerlos para que estén abiertos a ser responsables de su ejecución y, de esta forma, facilitar su implementación.

4. Implementar una estrategia de comunicación pensada, continua y evaluada para hacer los ajustes requeridos en el transcurso de su ejecución, para que al final, se tenga una toma de decisiones correcta y adecuada para el éxito de cualquiera de los logros propuestos.

5. Contar con recursos humanos especializados en materia de comunicación que permita poner en marcha las acciones, darle continuidad, y conseguir la implicación de todos los participantes en materia de comunicación institucional. A la luz de los cambios en el nivel de información, la percepción y la opinión se genere, se irá adecuando progresivamente la estrategia de comunicación.

Para West \& Turner (2005), los componentes de una estrategia de comunicación son los siguientes:

1. Visión general como punto de partida, visualizando la manera actual que se maneja la información que desea pasar y a las personas a quienes desea llegar.

2. Objetivos, para saber qué se desea lograr.

3. Mensaje, establecer que se quiere decir.

4. El público destinatario, determinar a quién se desea comunicar el mensaje

5. Consideraciones estratégicas, que permita establecer qué más debe tomar en cuenta al comunicar

6. Enfoque y actividades, para saber cómo se emitirá el mensaje.

7. Establecimiento de recursos, considerando financiamiento y personas.

8. Evaluación, para establecer si la estrategia funciona.

La estrategia de comunicación debe ser efectiva y medible como un factor importante para el éxito del negocio, por lo que se requiere convertir a la comunicación en un activo estratégico empresarial.

La evaluación de las estrategias de comunicación se dirige a verificar las consecuencias de la implementación de un programa y éstas suelen manifestarse de formas tan ambiguas e imprecisas que, en ocasiones, parece que no permiten su medición. De acuerdo a Scriven (1967) esta forma de evaluación recibe la denominación sumativa y describe si un programa o plan posee unos objetivos concretos, articulados y mensurables, antes de que éste sea implementado, por lo que resulta de gran ayuda en el proceso de toma de decisiones relativos a si continuar, o no, con el desarrollo del plan.

Asimismo, para llevar a cabo una medición evaluativa es evidente que debe realizarse, también, una investigación previa, de modo que se disponga de un elemento en relación al cual comparar los posibles logros alcanzados en la evaluación posterior. 
La forma en que se alcanzan los objetivos no forma parte de la evaluación sumativa, y hay que recurrir a la evaluación formativa, que es aquella que proporciona información de diagnóstico acerca de la mejor estrategia a implementar en el plan (Pavlic, 1999, pág. 84).

La gestión de la comunicación como una estrategia dentro de las empresas, es un elemento cada día más necesario y relevante dentro del sector empresarial. Como señala Herranz (2007), está probado que existe una relación directa entre la inversión realizada en gestionar la comunicación y la efectividad en la consecución de los objetivos de algunos programas.

En ocasiones, resulta paradójico pensar que muchas personas opinan y creen en la importancia de la comunicación, y, sin embargo, la realidad contrasta con los escasos recursos que se invierten para potenciarla. Quizás porque dentro del área de la comunicación hay que desterrar todavía algunas falsas ideas o prejuicios como que:

- La gestión de la comunicación es sólo un gasto y no una inversión.

- Gestionar la comunicación es caro porque se asocia sólo con la publicidad.

- La comunicación es poco rentable porque es difícil de cuantificar sus resultados.

- La comunicación se limita a editar una revista para el trabajador o socios que, en muchos casos, apenas se lee.

- La gestión de la comunicación precisa de abundantes recursos humanos y financieros.

- La formación en comunicación no es necesaria, todas las personas saben comunicarse.

Estas falsas ideas provocan la escasa utilización de este recurso por parte de las organizaciones.

Para Apolo y Báez (2017) las categorías que se deberán tomar en cuenta para gestionar la comunicación son: Identidad, imagen y reputación.

Cada una de estas categorías, para ser abordada debe responder a componentes específicos, los mismos que están compuestos por elementos propios, que en su conjunto brindarán datos relevantes para la medición y toma de decisiones que marcarán el diseño de investigación y posterior propuesta para el plan de gestión de Comunicación Corporativa.

El concepto de eficacia publicitaria es habitualmente utilizado para medir los resultados de una campaña publicitaria o un anuncio, aunque también es frecuente relacionarlo con el mensaje publicitario y el plan de medios. Sin embargo, la utilización de este término no se ha correspondido siempre con un mismo significado, debido al confusionismo que existe en relación con cuáles son los objetivos publicitarios y cómo deben medirse sus logros.

Tradicionalmente ha existido una tendencia a medirla en términos de ventas o de cambios de comportamiento del consumidor, olvidando que en la demanda y en el comportamiento del mercado intervienen, además de la publicidad, otros elementos de diferente índole tales como el precio, las promociones, las políticas comerciales de los competidores, etc. Una campaña 
ISSN: 2602-8085

publicitaria es eficaz en la medida en que cumple los objetivos para los cuales ha sido diseñada (Palacio \& Santana, 1996).

\section{Metodología}

La investigación es de tipo descriptivo, permitió llegar a conocer las situaciones, costumbres y actitudes predominantes a través de la descripción exacta de las actividades, objetos, procesos y personas. Su meta no se limita a la recolección de datos, sino a la predicción e identificación de las relaciones que existen entre dos o más variables. Los investigadores no son meros tabuladores, sino que recogen los datos sobre la base de una hipótesis o teoría, exponen y resumen la información de manera cuidadosa y luego analizan minuciosamente los resultados, a fin de extraer generalizaciones significativas que contribuyan al conocimiento (Morales, F., 2011).

Los métodos empleados son: analítico sintético, inductivo y deductivo. El enfoque de la investigación es cuali-cuantitativo, las investigaciones con enfoque cuantitativo usan la recolección y el análisis de información para contestar preguntas de investigación, así como comprobar hipótesis establecidas anteriormente, confía en la medición numérica, el conteo, y la utilización de estadísticas para establecer con precisión patrones en una población (Gómez, M., 2006).

Por otra parte, la investigación cualitativa aparece como una ventajosa forma de acercarse a comprender dicho conocimiento compartido, especialmente pues se rescata lo que los propios sujetos, en sus propias expresiones, identifica y señalan como sustancial a las experiencias que se investigan. Rescatando por tanto no la lectura de la realidad de parte del investigador, sino de los sujetos y las construcciones sociales que han realizado de su propia realidad. (Guajardo \& Castro, 2007)

La población, es el conjunto de todos los casos que concuerdan con una serie de especificaciones. Es decir, todos los individuos que forman parte de un conjunto delimitado por ciertas características comunes.

En el caso específico de la presente investigación la población estuvo conformada por los socios potenciales de la Cooperativa de Ahorro y Crédito "San Jorge" Ltda., quienes constituyen la población económicamente activa del cantón Riobamba. Dicha población de acuerdo con el Censo de Población y Vivienda (2010), son un total de 100.585

Con el objetivo de determinar la confiabilidad del instrumento aplicado se determinó el índice de confiabilidad Alfa Cronbach. 
El índice Alfa Cronbach trata de la consistencia interna que toma valores entre 0 y 1 y que sirve para comprobar si el instrumento que se está evaluando recopila información defectuosa y por tanto nos llevaría a conclusiones equivocadas o si se trata de un instrumento fiable que hace mediciones estables y consistentes (González \& Pazmiño, 2015).

Su interpretación radica que en cuanto más se acerque el índice al extremo 1, mejor es la fiabilidad, considerando una fiabilidad respetable a partir de 0,80 .

A continuación, se muestran los resultados obtenidos mediante el sistema SPSS, el cual muestra un resultado del Alfa de Cronbach de 0,812 mostrando una fiabilidad respetable de los resultados obtenidos en la encuesta.

Tabla 1. Resultados del Alfa de Cronbach

\section{Estadísticos de fiabilidad}

\begin{tabular}{|c|c|}
\hline Alfa de Cronbach & N de elementos \\
\hline, 812 & 15 \\
\hline
\end{tabular}

\section{Fuente: SPSS}

Para la determinación del tamaño de la muestra se consideró la población a analizar de 100.585, a través de la aplicación de la siguiente fórmula:

$$
n=\frac{N \sigma^{2} Z^{2}}{(N-1) e^{2}+\sigma^{2} Z^{2}}
$$

Dónde:

n: el tamaño de la muestra.

$\mathrm{N}$ : tamaño de la población $=100.585$

$\sigma$ : Desviación estándar de la población $=0,5$.

Z: niveles de confianza $=1.96$

e: Límite aceptable de error de la muestra $=0.05$

El resultado de la aplicación de la fórmula dio como resultado una muestra representativa de 383. 


\section{Resultados}

Tabla 2: Aspectos para seleccionar una institución financiera

\begin{tabular}{|c|c|c|}
\hline Respuestas & Frecuencia & $\%$ \\
\hline Tasas de interés & 124 & $22 \%$ \\
\hline Calidad del servicio & 97 & $17 \%$ \\
\hline Solvencia, seguridad y confianza & 154 & $28 \%$ \\
\hline Rapidez en los trámites & 103 & $19 \%$ \\
\hline Tipo de productos y servicios financieros ofertados & 53 & $10 \%$ \\
\hline Servicios electrónicos & 25 & $4 \%$ \\
\hline TOTAL & 556 & $100 \%$ \\
\hline
\end{tabular}

Fuente: Encuesta

Realizado por: Las autoras

Tabla 3: Conocimiento sobre la Cooperativa

\begin{tabular}{|c|c|c|}
\hline Respuestas & Frecuencia & $\%$ \\
\hline Siempre & 34 & $9 \%$ \\
\hline Alguna Vez & 225 & $59 \%$ \\
\hline Nunca & 124 & $32 \%$ \\
\hline TOTAL & 383 & $100 \%$ \\
\hline
\end{tabular}

Fuente: Encuesta

Realizado por: Las autoras

Tabla 4: Probabilidad de interesarse en los productos de la cooperativa

\begin{tabular}{lcc}
\multicolumn{1}{c}{ Respuestas } & Frecuencia & \% \\
\hline Bastante probable & 129 & $34 \%$ \\
Algo probable & 186 & $49 \%$ \\
Poco probable & 45 & $12 \%$ \\
Nada probable & 23 & $6 \%$ \\
\hline \multicolumn{1}{c}{ TOTAL } & $\mathbf{3 8 3}$ & $\mathbf{1 0 0 \%}$ \\
\hline
\end{tabular}

Fuente: Encuesta

Realizado por: Las autoras

Tabla 1: Frecuencia con que se utiliza las redes sociales

\begin{tabular}{lccc}
\hline & Respuestas & Frecuencia & \% \\
\hline Siempre & 275 & $72 \%$ \\
Alguna Vez & 92 & $24 \%$ \\
Nunca & 16 & $4 \%$ \\
\hline \multicolumn{2}{c}{ TOTAL } & $\mathbf{3 8 3}$ & $\mathbf{1 0 0 \%}$ \\
\hline
\end{tabular}

Fuente: Encuesta

Realizado por: Las autoras 
Tabla 2: Medios por los que se desea conocer las promociones

\begin{tabular}{|c|c|c|}
\hline Respuestas & Frecuencia & $\%$ \\
\hline Radio & 116 & $15 \%$ \\
\hline Televisión & 68 & $9 \%$ \\
\hline Prensa & 81 & $11 \%$ \\
\hline Gigantografías & 48 & $6 \%$ \\
\hline Redes Sociales & 285 & $38 \%$ \\
\hline Publicidad Móvil (Buses) & 119 & $16 \%$ \\
\hline Otros & 34 & $5 \%$ \\
\hline TOTAL & 751 & $100 \%$ \\
\hline
\end{tabular}

Fuente: Encuesta

Realizado por: Las autoras

\section{Discusión}

Entre los principales aspectos que se tienen en cuenta a la hora de seleccionar una institución financiera se encuentran la solvencia, seguridad y confianza, seguido por las tasas de interés y la rapidez en los tramites, la calidad del servicio y en menor medida los tipos de productos y servicios que se ofrecen y los servicios electrónicos. De forma general se observa a diferencia de otros mercados, una mayor valoración de la solvencia y seguridad de la institución sobre la variedad de productos que esta pueda ofrecer. Por lo que en estos elementos son en los que se debe priorizar las estrategias de comunicación.

Con respecto al nivel de conocimiento de la cooperativa, el 68\% de los encuestados exponen que han oído, visto o leído siempre o algunas veces a cerca de la Cooperativa de Ahorro y Crédito "San Jorge" Ltda., mostrando un mayor nivel de conocimiento de esta cooperativa con respecto a las demás.

Los resultados del nivel de interés de los encuestados en los productos y servicios que ofrece la cooperativa son altamente positivos.

El diagnóstico sobre el uso de las redes sociales en los encuestados muestra que casi la totalidad de los encuestados las utilizan evidenciando que este es uno de los medios en los que se debe realizar la estrategia de comunicación pues además en uno de los medios en los cuales más se recordó la publicidad de las instituciones financieras por parte de los encuestados.

Respecto a los medios en los que más se desea conocer las promociones según el criterio de la totalidad de los encuestados son las redes sociales, seguido de la publicidad en buses, por lo que en estos medios son en los que se deben enfocar la estrategia de comunicación. 


\section{Estrategias de comunicación}

Partiendo de los resultados del diagnóstico, se diseñan y ejecutan las siguientes estrategias:

1. Contratar un asesor experto en Microcrédito: Al contratar un asesor de microcrédito se prevé colocar capital en microcréditos, pues este representa el segundo producto en el que se mostraron interesados los encuestados. Con la incorporación de dicho producto se incrementa el beneficio para la institución, pues el spring financiero en este producto es mayor.

El asesor de microcrédito realizará un trabajo de campo el cual mediante una adecuada sectorización permitirá hacer un trabajo puerta a puerta en los sectores de interés de la Institución.

2. Contratar un Community Manager: Sus funciones radican el manejo de la página web y la actualización diaria de los perfiles de las redes sociales de la cooperativa. También se encarga de coordinar las respuestas adecuadas a las inquietudes de los clientes, así como realizar y dirigir las publicidades sobre la entidad en las redes virtuales; así como la retroalimentación a la empresa de las opiniones de los cibernautas. Una de sus labores más importantes es la de recopilar información acerca de las compañías que representan una competencia, para realizar campañas y estrategias en pro de la empresa.

3. Realizar el evento por aniversario institucional en un lugar céntrico de la ciudad de Riobamba: Con la finalidad de dar a conocer a la Institución a través de este tipo de eventos se propone realizar los eventos de aniversario en lugares estratégicos de la ciudad como: Plaza Eloy Alfaro, Parque Guayaquil o Plaza La Concepción.

\section{Estrategias varias}

- Disminuir el monto de apertura de cuenta de \$100,00 a \$ 35,00 dólares mínimo esto permitirá captar mayor número de socios. El valor que falta por completar en certificados de aportación de $\$ 65,00$ se los deberá diferir para 6 meses permitiendo que el socio complete este valor después de la apertura de cuenta.

- En el caso de socios que aperturen la cuenta para realizar créditos para dar cumplimiento a lo que estipula el Manual de Créditos y Cobranzas en donde consta que para acceder a créditos se debe tener el valor total de certificados de aportación se puede abrir la cuenta con $\$ 35,00$ y el valor de $\$ 65,00$ para completar certificados de aportación se financiará del monto del crédito.

- Realizar visitas personalizadas a los sectores de interés de la Cooperativa para dar a conocer los productos, servicios y beneficios institucionales.

- Incentivar la apertura de cuentas entregando regalos por hacerse socios de la Institución.

- Diseñar trípticos con información general de la Cooperativa. Diseñar dípticos con información específica de microcréditos. 
- Establecer una comunicación adecuada mediante redes sociales, crear la fan page de Facebook en donde se dará a conocer los productos, servicios y beneficios institucionales.

- Diseñar la imagen corporativa de la institución para posicionarla en la mente de sus socios.

- Auspicio en eventos deportivos y culturales de interés social.

- Publicidad móvil colocada en dos buses de la Cooperativa Liribamba la cobertura de estos buses es de 30 paradas que cubren toda la ciudad.

- Valla publicitaria ubicada en el terminal Inter-cantonal.

- Publicidad radial en medios de comunicación masiva, radio, prensa y televisión para difundir todo lo relacionado al evento por aniversario.

- Incluir en la Planificación Estratégica 2018 - 2021 una perspectiva relacionada con comunicación integral interna y externa

Las estrategias diseñadas y ejecutadas, constituyeron un apoyo efectivo para la cooperativa evidenciando resultados positivos que se detallan a continuación:

En el caso particular de socios captados, se observa un crecimiento del 51,70\% en el número de socios captados desde enero a junio de 2019 en comparación con el mismo periodo del 2018. En el caso particular de los clientes se evidencia igualmente un crecimiento del $130,77 \%$ en el 2019 en comparación con el mismo periodo del 2018.

Respecto a las colocaciones de consumo se muestra igualmente un crecimiento del $34,01 \%$ siendo desde enero a junio de 2019 de 2.972.926,47 USD mientras que en el mismo periodo del 2018 se ha llegado a 3.984.004,80 USD.

Figura 1: Colocaciones de Microcrédito periodo 2018-2019

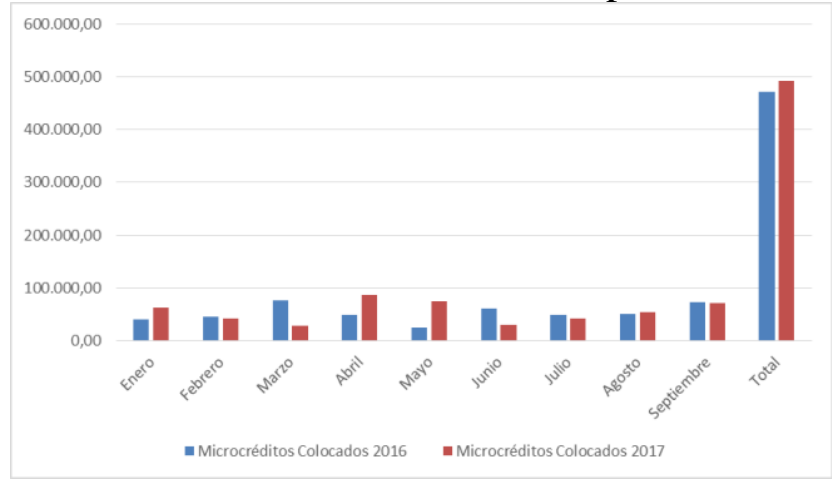

Fuente: Cooperativa de Ahorro y Crédito "San Jorge" Ltda. 
ISSN: 2602-8085

En cuanto a las colocaciones de microcrédito igualmente se incrementan en un 4,28\%

De forma general se evidencia un mejor resultado luego de la implementación de las estrategias de comunicación, alcanzándose una mejor captación de socios, así como colocaciones en consumo y microcrédito.

A modo de resumen se comprueba además la relación entre las variables, plan de Comunicación y Posicionamiento. Dicha comprobación se realiza mediante el coeficiente de correlación de Spearman tomando como base las interrogantes 2 y 4 de la encuesta aplicada a los clientes potenciales, los cuales muestran los resultados de los planes de comunicación y el posicionamiento de la empresa respectivamente.

El coeficiente de correlación de Spearman es una medida adecuada en el caso de variables en escala ordinal y sus resultados oscilan entre -1 y 1 , siendo clasificada la relación de acuerdo con los criterios de interpretación que se muestran en la siguiente tabla (Pedrosa \& Dicovsky, 2006, p. 58).

Tabla 8: Niveles de Correlación del Coeficiente Spearman

\begin{tabular}{cc}
\hline $\begin{array}{c}\text { Valor del coeficiente } r \\
\text { (positivo o negativo) }\end{array}$ & Significado \\
\hline 0,00 a 0,19 & Correlación muy débil \\
0,20 a 0,39 & Correlación débil \\
0,40 a 0,69 & Correlación moderada \\
0,70 a 0,89 & Correlación fuerte \\
0,90 a 1,00 & Correlación muy fuerte \\
\hline
\end{tabular}

Fuente: (Ceroni, 2013)

A continuación, se muestran los resultados de la correlación entre las variables mostrando un resultado de 0,842 siendo esta una correlación fuerte positiva, lo que significa que el plan de comunicación tiene una influencia fuerte sobre el posicionamiento de la Cooperativa de Ahorro y Crédito "San Jorge" Ltda. 
Tabla 9: Resultados de la correlación de Variables

\begin{tabular}{|c|c|c|c|c|}
\hline \multicolumn{5}{|c|}{ Correlaciones } \\
\hline & & & $\begin{array}{c}\text { Plan de } \\
\text { Comunicación }\end{array}$ & Posicionamiento \\
\hline \multirow{6}{*}{$\begin{array}{l}\text { Rho de } \\
\text { Spearman }\end{array}$} & Plan de Comunicación & Coeficiente de correlación & 1,000 & $842^{\prime}$ \\
\hline & & Sig. (bilateral) & &, 000 \\
\hline & & $\mathrm{N}$ & 383 & 383 \\
\hline & Posicionamiento & Coeficiente de correlación & $842^{* *}$ & 1,000 \\
\hline & & Sig. (bilateral) &, 000 & . \\
\hline & & $\mathrm{N}$ & 383 & 383 \\
\hline
\end{tabular}

**. La correlación es significativa al nivel 0,01 (bilateral).

\section{Conclusiones}

Fuente: SPSS

- Se observa que, a diferencia de otros mercados, existe una mayor valoración de la solvencia y seguridad de la institución sobre la variedad de productos que esta pueda ofrecer. Por lo que en estos elementos son en los que se prioriza las estrategias de comunicación.

- Con respecto al nivel de conocimiento de la cooperativa, el 68\% de los encuestados exponen que han oído, visto o leído siempre o algunas veces a cerca de la Cooperativa de Ahorro y Crédito "San Jorge" Ltda., mostrando un mayor nivel de conocimiento de esta cooperativa con respecto a las demás.

- Solamente el $40 \%$ de los encuestados conocen que la Cooperativa de Ahorro y Crédito "San Jorge" Ltda., es una entidad de carácter abierto lo cual le permite captar socios de toda la comunidad

- Se trazaron estrategias como la contratación de un experto en microcrédito, así como de un community manager, se realizó una feria de emprendimientos que permitió difundir las actividades productivas de los asociados, además se ha diseñado trípticos y dípticos con información institucional, se ha creado la página institucional de la cooperativa en Facebook la cual ya ha alcanzado un total de 3350 amigos y se diseñó la papelería institucional de la cooperativa

- De forma general se evidencia un mejor resultado luego de la implementación de las estrategias de comunicación, alcanzándose un incremento del 51,70\% en captación de socios, así como un crecimiento del $34,01 \%$ en colocaciones de consumo y de un $4,28 \%$ en microcrédito.

\section{Referencias bibliográficas}

Apolo, D. \& Báez, V. (2017). Gestión de Comunicación Corporativa: consideraciones para el abordaje de su estudio y práctica. Revista Latina de Comunicación Social, 521 539 . 
Bartolie, A. (2013). Comunicación y Organización (6ta ed.). Madrid: Paidós.

Gómez, M. (2006). Introduccion a la metodologia de la investigación científica. Argentina: Publicación de Editorial Brujas de Córdova - Argentina.

González, A. \& Pazmiño, M. (2015). Cálculo e interpretación del Alfa de Cronbach para el caso de validación de la consistencia interna de un cuestionario, con dos posibles escalas tipo Likert.

Guajardo, S., \& Castro. (2007). La investigación cualitativa, una discusión presente. Obtenido de la investigación cualitativa, una discusión presente: http://www.scielo.org.pe/scielo.php?script=sci_isoref\&pid=S172948272007000100008\&lng=es\&tlng=es

Herranz, J. (2007). La gestión de la comunicación como elemento generador de transparencia en las organizaciones no lucrativas. CIRIEC.

Kotler, P. (2012). Estrategias para la atracción de clientes y negocios en Latinoamérica. México: Pearson Prentice Hall.

West, R., \& Turner, L. (2005). Teoría de la Comunicación. Madrid: McGraw Hill

Morales, E., \& Soler. (2008). La planificación de la comunicación empresarial. Barcelona: Universidad Autónoma de Barcelona.

Orejuela, S. (2011). Análisis de la comunicación en la gestión de la Responsabilidad Social Empresarial en Venezuela. Caracas: UEN.

Palacio, B., \& Santana, M. (1996). Técnicas de medición de la eficacia publicitaria. Barcelona: Ariel Economía.

Pavlic. (1999). La investigación en Relaciones Públicas. Barcelona: Gestión 2000.

Regouby, C. (2014). La Comunicación Global (4ta ed.). Barcelona: McGraw-Hill.

Scriven. (1967). The Methodology of Evaluation. Rand McNally: Chicago 


\section{PARA CITAR EL ARTÍCULO INDEXADO.}

Pazmiño Garzón, D., Moncayo Sánchez, Y., Carrión Torres, M., \& Cuadrado Samaniego, V. (2019). Estrategias de comunicación para mejorar el posicionamiento de la cooperativa de ahorro y crédito "San Jorge" Ltda. Ciencia Digital, 3(3.3), 44-58. https://doi.org/10.33262/cienciadigital.v3i3.3.769

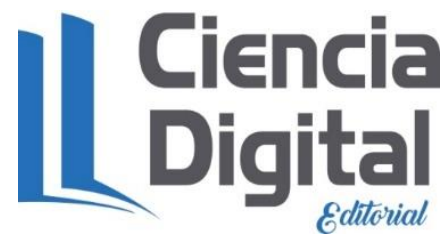

El artículo que se publica es de exclusiva responsabilidad de los autores y no necesariamente reflejan el pensamiento de la Revista Ciencia Digital.

El artículo queda en propiedad de la revista y, por tanto, su publicación parcial y/o total en otro medio tiene que ser autorizado por el director de la Revista Ciencia Digital.
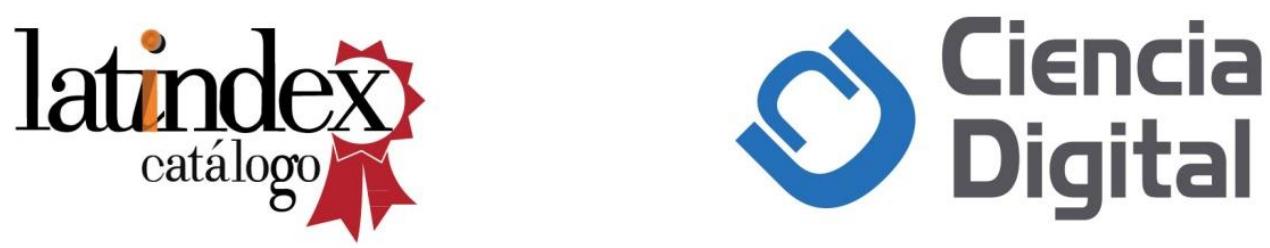